\title{
A low complexity high efficiency hybrid multiplicative-additive crest factor reduction for OFDM systems
}

\author{
Khalid Al-Hussaini ${ }^{1}$ - Borhanuddin M. Ali ${ }^{2}$ - Pooria Varahram ${ }^{3}$. \\ Shaiful Jahari Hashim ${ }^{1}$ - Ronan Farrell ${ }^{3}$
}

Published online: 27 September 2016

(C) Springer Science+Business Media New York 2016

\begin{abstract}
In this paper, we propose a novel technique to reduce the crest factor $(\mathrm{CF})$ in orthogonal frequency division multiplexing systems. It consists of two inverse fast Fourier transform (IFFT) blocks, the input symbols of the first IFFT are the mapped symbols, whereas the input symbols of the second IFFT are the summations of the absolute value of the real part of the outer signal constellation points and zero symbols. First, the output of the two IFFT blocks is partitioned into four subblocks, which are subsequently used to rearrange the subblocks with padding zeros in a specific manner. Then, a new optimization scheme is introduced, in which only a single two-phase sequence and four iterations need to be applied. Numerical analysis shows that the proposed hybrid technique achieves better $\mathrm{CF}$ reduction performance with significantly lower complexity and better bit error rate performance than
\end{abstract}

\footnotetext{
Khalid Al-Hussaini

khalid00alhussaini@gmail.com

Borhanuddin M. Ali

borhan@upm.edu.my

Pooria Varahram

pooria.varahram@nuim.ie

Shaiful Jahari Hashim

sjh@upm.edu.my

Ronan Farrell

rfarrell@eeng.nuim.ie

1 Department of Computer and Communications Systems Engineering, Universiti Putra Malaysia, 43400 UPM Serdang, Selangor, Malaysia

2 Department of Computer and Communications Systems Engineering,Research Centre of Excellence for Wireless and Photonic Networks (WiPNET), Universiti Putra Malaysia, 43400 UPM Serdang, Selangor, Malaysia

3 CTVR - The Telecommunication Research Centre, Callan Institute, Department of Electronic Engineering, National University of Ireland, Kildare, Ireland
}

the existing scrambling (multiplicative) and additive $\mathrm{CF}$ techniques.

Keywords OFDM $\cdot$ Crest factor $\cdot$ PAPR $\cdot$ PTS $\cdot$ TR

\section{Introduction}

Transmitting digital signals with high data rates using single carrier results in a lot of difficulties because of the multipath propagation. Due to the short symbol time and the long channel response time for it, this results in very high requirements for the equaliser. Dividing the data rate into $N$ subcarriers results in an $N$ times longer symbol duration. In spite of this improvement, there are still some inter-symbol-interferences due to the different runtimes of the signals in the multipath environment $[1,2]$. To reduce the inter-symbol interference a guard interval is introduced. This guard interval provides some time for the symbols to raise and decay. One of the most advantages is the abdication of complex filter banks due to the use of digital signal processing. A characteristic of an orthogonal frequency division multiplexing (OFDM) system is the equidistant subchannel order. The distance between different subchannels is chosen in a way that they do not disturb each other $[2,3]$.

From the basic principles of OFDM, we know that in the frequency and time selective transmission environment, the channel does not change significantly in one OFDM symbol or one OFDM subcarrier, however, it changes from subcarrier to subcarrier in the frequency domain and symbol to symbol in the time domain [4]. When the channel has a deep fading, some subcarriers, and some OFDM symbols will suffer from strong noise interference, which causes a degrading signalto-noise ratio (SNR) at these positions resulting in excessive burst errors at the receiver. To overcome this problem, coding and interleaving are employed in OFDM system [5-7]. 
One of the major disadvantages of OFDM systems is its crest factor $(\mathrm{CF})$ in the time domain. This is because there are many subcarriers are in phase with each other and each of the subcarrier having different phase values. When all subcarriers are added up together, it will lead to a sudden shot up in output envelope as subcarriers attain peak value simultaneously. Therefore, the contrast between the peak value and average value in an OFDM system might be very high. The large CF causes the transmit power amplifier to enter the non-linear region, distorting the signal and resulting in a significant increase in the bit error rate (BER) at the receiver. Clearly, it is important that the CF should be reduced to ensure efficient transmissions in OFDM systems $[1,3,4]$.

Numerous $C F$ reduction techniques have been proposed to reduce the CF. These techniques can be classified into signal scrambling (multiplicative) techniques such as selective level mapping (SLM) and partial transmit sequences (PTS) $[5,6,8]$ and additive techniques such as tone reservation (TR), tone injection (TI), peak cancellation, and clipping and filtering $[1,9,10]$.

In this paper, we propose a hybrid multiplicative-additive $\mathrm{CF}$ reduction technique for OFDM Systems. This technique uses two IFFT blocks and a new optimization scheme in which only 4 iterations and a single two-phase sequence need be applied. The simulation results indicate that the performance of the proposed technique improves $\mathrm{CF}$ reduction, BER performance and reduces the computational complexity by up to $33 \%$ than the existing scrambling (multiplicative) and additive $\mathrm{CF}$ techniques. Moreover, no side information is needed, allowing for an increased transmission efficiency.

\section{System model}

In OFDM systems, the data stream with rate $\mathrm{R}$ bps is mapped to phase shift keying (PSK) or quadrature amplitude modulation (QAM). A set of $N$ mapped signal is converted to $N$ parallel streams by way of a serial-to-parallel converter. These sets are known as the OFDM symbol. Afterward, the IFFT with length $N$ is used to produce orthogonal data subcarriers. Then, all orthogonal subcarriers are transmitted simultaneously over the symbol interval $T$. The complex baseband OFDM signal $x(t)$ with $N$ orthogonal subcarriers can be written as $[1,4]$.

$x(t)=\frac{1}{\sqrt{N}} \sum_{k=0}^{N-1} X_{k} e^{j 2 \pi k \Delta f t}$

where $\Delta f=\frac{1}{T}$ is the subcarrier spacing, $X_{k}$ is the $k_{t h}$ frequency domain signals in OFDM.

We can describe the characteristics of the power in terms of their magnitudes as follows:
$C F=\sqrt{P A P R}$

where PAPR is the peak-to-average power ratio. The PAPR of the signal is defined as [3]:

$\operatorname{PAPR}(x(t))=\frac{\max _{0 \leq t \leq T}|x(t)|^{2}}{E\left[|x(t)|^{2}\right]}$

where $E[$.$] is the expectation value operator.$

The high peaks appear when $N$ different mapped symbols phases in (1) are accumulated constructively [2]. Generally, the complementary cumulative distribution function (CCDF) is used to capture the statistical PAPR properties of the PAPR in OFDM system, and is defined as:

$C C D F\left(P A P R_{0}\right)=\operatorname{Pr}\left\{P A P R>P A P R_{0}\right\}$

where $P A P R_{0}$ is a constant.

The most attractive conventional multiplicative and Additive CF reduction techniques are PTS and TR, respectively. Both techniques are described below.

\subsection{Tone reservation technique}

In the TR technique, the transmitter and receiver know the set of data-carrying subcarriers [5]. Different methods have been proposed by researchers to construct the reduction signals. However, the generation methods of these signals are all iterative and may sometimes require tens of loops $[1,9]$.

The TR technique can reduce the PAPR value by utilizing the reserved subcarriers, which are not used for data transmission. Based on the TR technique, the baseband signal in (1), can be expressed as:

$\hat{x}(t)=x(t)+r(t)=\frac{1}{\sqrt{N}} \sum_{k=1}^{N-1}\left(X_{k}+R_{k}\right) e^{j 2 \pi k \Delta f t}$

where $r(t)\left(\mathbf{r}=\left[r_{0}, r_{1}, \ldots, r_{N-1}\right]^{T}\right)$ is the peak-canceling signal in time domain, and $\mathbf{R}=\left[R_{0}, R_{1}, \ldots, R_{N-1}\right]^{T}$ is the peak-canceling signal vector in frequency domain, which is generated by using $Q$ peak reduction tones (PRTs). The $Q$ PRTs do not carry any data information, and they are only used for reducing the PAPR.

The modulated data signal $X_{k}$ and the peak reduction signal $R_{k}$ are restricted to stand in disjoint subcarrires tones as shown in Fig. 1, that is:

$X_{k}+R_{k}= \begin{cases}X_{k}, & \text { if } k \in K^{c} \\ R_{k}, & \text { if } k \in K\end{cases}$

where $K=\left\{k_{0}, k_{1}, \ldots, k_{Q-1}\right\}$ is the dummy data signal (PRTs) on the $k$ th subcarrier, and $K^{c}$ is the set of the remain- 


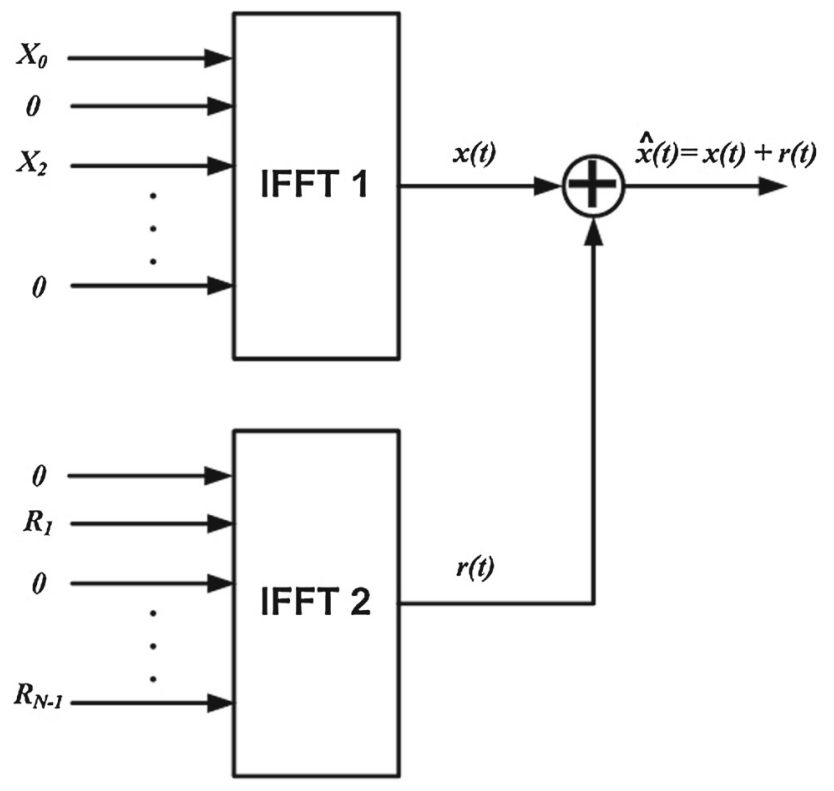

Fig. 1 Block diagram of the TR technique

ing subcarriers in $N$ used for the modulated data signal. However, $R_{k} \neq 0$ only if $k \in K$. $\mathbf{r}$ is designed to minimize the maximum value of $\hat{x}(t)$. A minimax CF optimization problem is defined as:

$\mathbf{r}^{\text {opt }}=\underbrace{\arg \min }_{r}\|\hat{x}\|_{\infty}$

where $\|.\|_{\infty}$ is the $\infty$-norm of a vector.

Equation (6) can be reconstructed as a quadratically constrained quadratic program (QCQP) problem, which is very complex. However, there are several TR based techniques that reduces complexity, for example, a simple suboptimal technique to define the value of $r$ is the iterative clipping and filtering (ICF). However, the main problem with the TR technique is its complexity and the receiver must know about the positions of the reserved subcarriers and disregards these signals.

\subsection{Partial transmit sequence technique}

In the conventional PTS (C-PTS) technique, the incoming serial random data vectors at the transmitter are mapped into QAM symbols and then converted from serial to parallel streams:

$\mathbf{X}=\left[X_{0}, X_{1}, \ldots, X_{N-1}\right]^{T}$

Then, $\mathbf{X}$ is partitioned into $M$ disjoint subblocks as shown in Fig. 2, which are represented by the vectors $\mathbf{X}_{m}(1 \leq m<$ $M$ ) of length $V$, where $N=M V$ for integers $M$ and $V$. For $m=1, \cdots, M$, let the matrix $\mathbf{A}$ be a zero-padded matrix of $\mathbf{X}_{m}$, which can be written as:

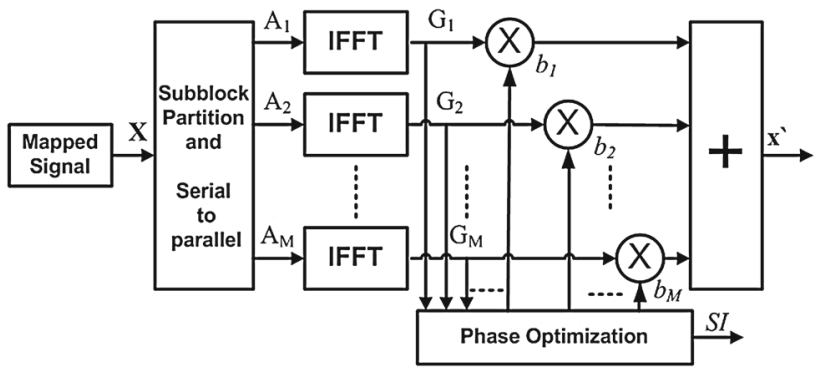

Fig. 2 Block diagram of the PTS technique

$\mathbf{A}=\left[\begin{array}{cccc}A_{11} & A_{12} & \cdots & A_{1, M} \\ A_{21} & A_{22} & \cdots & A_{2, M} \\ \vdots & \vdots & \ddots & \vdots \\ A_{L N, 1} & A_{L N, 2} & \cdots & A_{L N, M}\end{array}\right]$

where $L$ is the oversampling factor. Then, let the matrix $\mathbf{G}$ be the zero-padded IFFT of $\mathbf{A}$, which can be written as:

$\mathbf{G}=\left[\begin{array}{cccc}G_{11} & G_{12} & \cdots & G_{1, M} \\ G_{21} & G_{22} & \cdots & G_{2, M} \\ \vdots & \vdots & \ddots & \vdots \\ G_{L N, 1} & G_{L N, 2} & \cdots & G_{L N, M}\end{array}\right]$

Next, the time domain sequences are combined to minimize the PAPR, this is done by applying the complex phase rotation factors $\mathbf{b}=\left[b_{1}, b_{2}, \ldots, b_{M}\right]^{T}$. The resulting time domain signal after combination can be written as:

$\mathbf{x}^{\prime}=\mathbf{G b}$

where $\mathbf{x}^{\prime}=\left[x_{1}^{\prime}, x_{2}^{\prime}, \ldots, x_{L N}^{\prime}\right]$ is the block of optimized signal samples. Hence, the objective of PTS is to come out with an optimal phase factor for the subblock set that minimizes the PAPR. The objective of the optimization problem is to identify optimum phases $\hat{\mathbf{b}}$ that satisfy:

$\left\{\hat{b}_{1}, \hat{b}_{2}, \ldots, \hat{b}_{M}\right\}=\underbrace{\arg \min }_{\left\{b_{1}, b_{2}, \ldots, b_{M}\right\}}(\underbrace{\max }_{1 \leq k<L N}\left|\sum_{m=1}^{M} b_{m} G_{k, m}\right|)$

where $b_{m} \in\{ \pm 1, \pm j\}$ and $(W=4)$, where $W$ is the number of phase weight factors. $b_{1}$ can be set equal to 1 without loss of performance $[1,3]$. Therefore, in the PTS technique, it is necessary to test $W^{M-1}$ sets of distinct possible candidate vectors b to satisfy (11). Accordingly, the computational complexity of the PTS technique increases exponentially with $M$. 
At the receiver, after the $N$-point fast Fourier transform (FFT), the frequency domain sequence can be written as:

$\mathbf{A}^{\prime}=F F T\left(\mathbf{x}^{\prime}\right)$

Then, the vector $\mathbf{A}^{\prime}$ is partitioned into $M$ disjoint subblocks, which are represented by the vector $\mathbf{A}^{\prime}{ }_{m}(1 \leq m<M)$ of length $V$, where $N=M V$ for certain integers $M$ and $V$. For $m=1, \ldots, M$, let the matrix $\hat{\mathbf{A}}$ be the zero-padded of $\mathbf{A}^{\prime}{ }_{m}$, which can be written as:

$\hat{\mathbf{A}}=\left[\begin{array}{cccc}\hat{A}_{11} & \hat{A}_{12} & \cdots & \hat{A}_{1, M} \\ \hat{A}_{21} & \hat{A}_{22} & \cdots & \hat{A}_{2, M} \\ \vdots & \vdots & \ddots & \vdots \\ \hat{A}_{N, 1} & \hat{A}_{N, 2} & \cdots & \hat{A}_{N, M}\end{array}\right]$

So, using the inverse phase rotation vector $\mathbf{b}$, we can recover the signal as follows:

$\hat{\mathbf{X}}=\hat{\mathbf{A}} \mathbf{b}$

As noted, in the PTS technique, only the phase information is changed. Accordingly, no out-of-band radiation occurs. The main problems in the PTS technique are that the complexity and it needs side information (SI) required $\left(\left\lfloor\log _{2} W^{M-1}\right\rfloor\right)$ by the receiver $[1,9]$.

\section{Proposed technique analysis}

In the proposed technique, the input symbols of the first IFFT are the mapped symbols, whereas the input symbols of the second IFFT are the summations of the absolute value of the real part of the outer signal constellation points and zero symbols. First, the output of the two IFFT blocks is partitioned into four subblocks, which are subsequently used to rearrange the subblocks with padding zeros in a specific manner. Then, a new optimization scheme is introduced, in which only a single two-phase sequence $\{0,1\}$ needs to be applied. After summation, the original constellation points are shifted to the right or left based on the weighting phase. If the weighting phase is 1 , then the original constellation is shifted to the right with changes to the sign of the phase of constellation points. If the weighting phase is 0 , then the original constellation is shifted to the left without changes to the sign of the phase of constellation points, and also the phase of the output of the first IFFT is rotated when the weighting phase is 1. The proposed technique does not require extra subcarriers for PRTs.

\subsection{Transmitter side of the Proposed technique}

At the transmitter of the proposed technique, as shown in Fig. 3, the incoming serial random data vector is initially mapped into QAM symbols and then converted from a serial stream to a parallel stream, such that:

$\mathbf{X}=\left[X_{0}, X_{1}, \ldots, X_{N-1}\right]^{T}$

where $N$ is the number of points of the IFFT. As explained in algorithm 1, when $\mathbf{X}$ passes through the first IFFT block, the $N$-point IFFT output can be expressed a follows:

$\mathbf{x}=\left[x_{0}, x_{1}, \ldots, x_{N-1}\right]^{T}$

Then, we calculate $C$ as follows:

$C=|\Re\{\hat{C}\}| \times N$

where $\hat{C}$ denote the maximum value of the real part of the outer signal constellation points, it works as anti-peak signal.

For example, in case of 4-QAM as shown in Fig. 3, $C$ can be calculated as follows:

$C=\sum_{n=0}^{N-1}\left|\Re\left\{X_{n}\right\}\right|$

Then, inserting $C$ to the first point of the second $N$-point IFFT, and the rest of points are all zeros, the output of second $N$-point IFFT can be derived as follows:

$\mathbf{c}=\left[c_{0}, c_{1}, \ldots, c_{N-1}\right]^{T}$

where $c_{0}=c_{1}=\ldots=c_{N-1}$. Then, the output of the first and second $N$-point IFFT are partitioned into four subblocks. Then, the subblocks are rearranged with padding zeros, as shown in Fig. 3. By using this combination, this signal can be reconstructed easily at the receiver. The output can be expressed as a matrix with size $4 \times N$. Each row in the following matrix is referred to as a subblock:

$\mathbf{d}_{4 \times N}=\left(\begin{array}{cccc}x_{0, \ldots, k-1} & 0_{0, \ldots, k-1} & c_{0, \ldots, k-1} & c_{k, \ldots, 2 k-1} \\ 0_{0, \ldots, k-1} & x_{k, \ldots, 2 k-1} & 0_{0, \ldots, k-1} & 0_{0, \ldots, k-1} \\ 0_{0, \ldots, k-1} & 0_{0, \ldots, k-1} & x_{2 k, \ldots, 3 k-1} & 0_{0, \ldots, k-1} \\ c_{2 k, \ldots, 3 k-1} & c_{3 k, \ldots, 4 k-1} & 0_{0, \ldots, k-1} & x_{3 k, \ldots, 4 k-1}\end{array}\right)$

where $k=(N / 4), x_{0, \ldots, n-1}$ are $k$ th-1 samples of the first IFFT, $c_{0, \ldots, n-1}$ are $k$ th- 1 samples of the second IFFT and $0_{0, \ldots, n-1}$ are $k$ th- 1 padding zeros.

The aim is to reduce the PAPR of the signal $\mathbf{x}$ such that $\operatorname{PAPR}(x+c)<\operatorname{PAPR}(x)$ by way of the following optimization scheme, while keeping the average power nearly unchanged. 
Fig. 3 Block diagram of the proposed technique

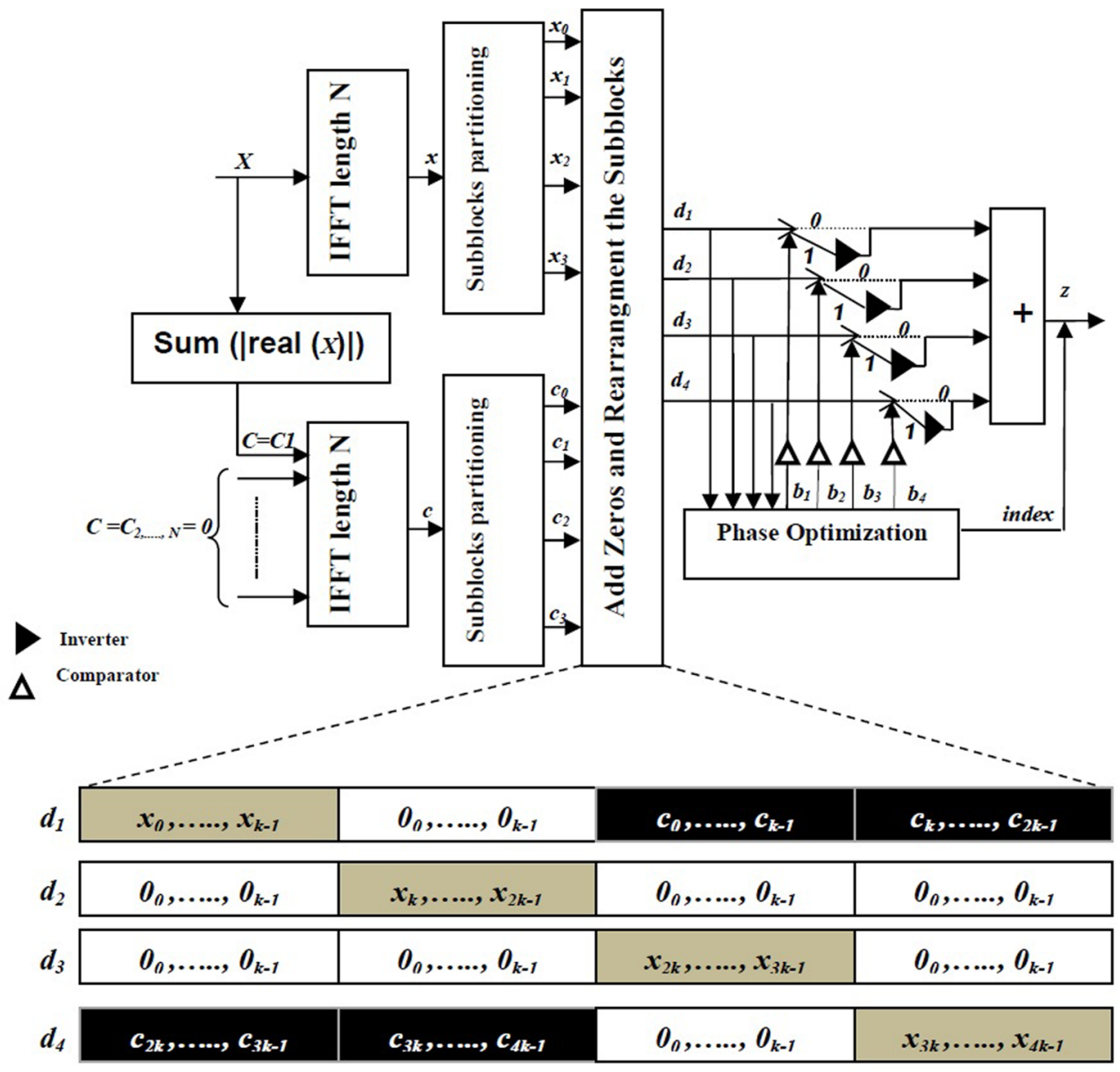

Then, we applied the optimization schemes in [4], but with $M=4$. Only two phase sequences $\{0,1\}$ are required. First, all phase sequence possibilities are generated using a $16 \times 4$ encoder $\mathbf{E}$ (i.e. $\left\{\begin{array}{llll}b_{1} & b_{2} & b_{3} & b_{4}\end{array}\right\}=\{0000,0001,0010,0011$, 0100, 0101, 0110, 0111,1000,1001, 1010, 1011, 1100, 1101, $1110,1111\}$ ), as shown in Table 1 . Then, the phase of each subblock is converted based on the proposed weight of phase rotation, as follows:

$\mathbf{z}=\sum_{m=1}^{4}(-1)^{b_{m}} \mathbf{d}_{m}$

where $b_{m} \in\{0,1\}, m$ is the row number of the matrix $\dot{\mathbf{x}}_{4 \times N}$ in (20).

As shown in Fig. 3, the comparator will detect whether the phase factor is 0 or 1 . If the weight of the phase fac- tor is 0 , the phase of elements of subblock does not change and passes directly to the summation unit. If the weight of the phase factor is 1 , the phase is rotated when passing through the inverter and then passed to the summation unit. As the first step, the PAPR of the combined signal can be calculated. For example, we check if the PAPR at $b_{1}=1$ is the lowest. Then, all phase sequences with $b_{1}=0$ will be omitted (i.e. $\left\{\begin{array}{llll}b_{1} & b_{2} & b_{3} & b_{4}\end{array}\right\}=\{0000,0001,0010,0011$, 0100, 0101, 0110, 0111\}). Thus, half of these sequences of Table 1 is omitted. Afterward, we check $b_{2}$ of the rest of the phase sequences (i.e. $\left\{\begin{array}{llll}b_{1} & b_{2} & b_{3} & b_{4}\end{array}\right\}=\{1000,1001,1010$, $1011,1100,1101,1110,1111\})$. If the PAPR at $b_{2}=1$ is the lowest, then all phase sequences with $b_{2}=0$ will be omitted (i.e. $\left.\left\{b_{1} b_{2} b_{3} b_{4}\right\}=\{1000,1001,1010,1011\}\right)$. Thus, half of these sequences are omitted. Afterward, we check $b_{3}$ of the rest of the phase sequences (i.e. $\left\{b_{1} b_{2} b_{3} b_{4}\right\}=\{1100$, 
Table 1 Candidate Phase Sequences using an $16 \times 4$ Encoder (E)

\begin{tabular}{|c|c|c|c|c|}
\hline Index & 1 & 2 & 3 & 4 \\
\hline$\left\{\begin{array}{llll}b_{1} & b_{2} & b_{3} & b_{4}\end{array}\right\}$ & $\left\{\begin{array}{llll}0 & 0 & 0 & 0\end{array}\right\}$ & $\left\{\begin{array}{llll}0 & 0 & 0 & 1\end{array}\right\}$ & $\left\{\begin{array}{llll}0 & 0 & 1 & 0\end{array}\right\}$ & $\left\{\begin{array}{llll}0 & 0 & 1 & 1\end{array}\right\}$ \\
\hline Index & 5 & 6 & 7 & 8 \\
\hline$\left\{\begin{array}{llll}b_{1} & b_{2} & b_{3} & b_{4}\end{array}\right\}$ & $\left\{\begin{array}{llll}0 & 1 & 0 & 0\end{array}\right\}$ & $\left\{\begin{array}{llll}0 & 1 & 0 & 1\end{array}\right\}$ & $\left\{\begin{array}{llll}0 & 1 & 1 & 0\end{array}\right\}$ & $\left\{\begin{array}{llll}0 & 1 & 1 & 1\end{array}\right\}$ \\
\hline Index & 9 & 10 & 11 & 12 \\
\hline$\left\{\begin{array}{llll}b_{1} & b_{2} & b_{3} & b_{4}\end{array}\right\}$ & $\left\{\begin{array}{llll}1 & 0 & 0 & 0\end{array}\right\}$ & $\left\{\begin{array}{llll}1 & 0 & 0 & 1\end{array}\right\}$ & $\left\{\begin{array}{llll}1 & 0 & 1 & 0\end{array}\right\}$ & $\left\{\begin{array}{llll}1 & 0 & 1 & 1\end{array}\right\}$ \\
\hline Index & 13 & 14 & 15 & 16 \\
\hline$\left\{b_{1} b_{2} b_{3} b_{4}\right\}$ & $\left\{\begin{array}{llll}1 & 1 & 0 & 0\end{array}\right\}$ & $\left\{\begin{array}{llll}1 & 1 & 0 & 1\end{array}\right\}$ & $\left\{\begin{array}{llll}1 & 1 & 1 & 0\end{array}\right\}$ & $\left\{\begin{array}{llll}1 & 1 & 1 & 1\end{array}\right\}$ \\
\hline
\end{tabular}

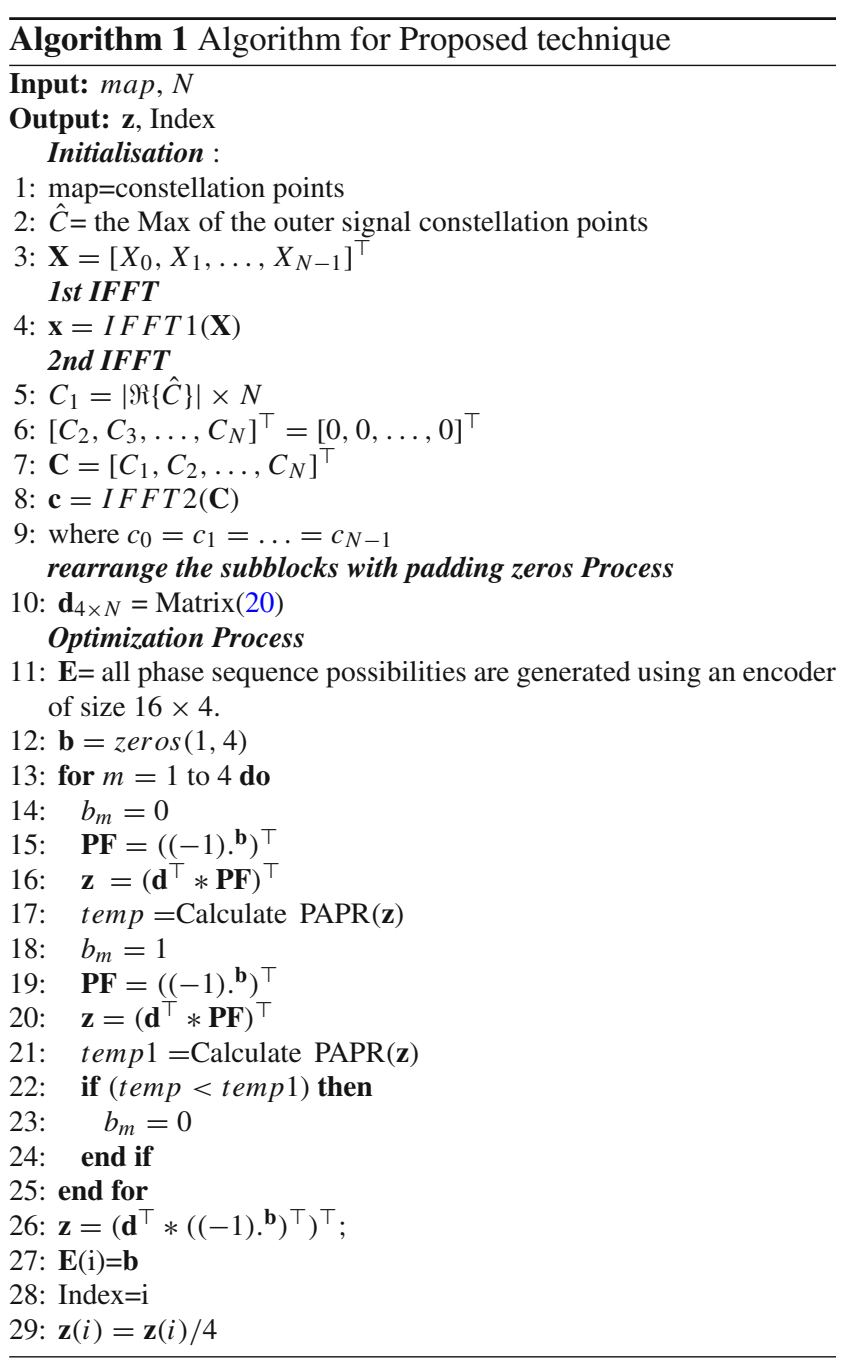

$1101,1110,1111\})$. If the PAPR at $b_{3}=1$ is the lowest, then all phase sequences with $b_{3}=0$ will be omitted (i.e. $\left.\left\{\begin{array}{llll}b_{1} & b_{2} & b_{3} & b_{4}\end{array}\right\}=\{1100,1101\}\right)$. Finally, one of the last two sequences (i.e. $\left.\left\{\begin{array}{llll}b_{1} & b_{2} & b_{3} & b_{4}\end{array}\right\}=\{1110,1111\}\right)$ will be the final candidate for the best sequence giving minimum PAPR. Then, the best sequence will be converted to an index, as shown in Table 1. For example, if the best sequence is $\{1110\}$, it will be converted to its index, which is 15 as shown in Table 1 . Then, we minimize the sample number 15 among $N$ -

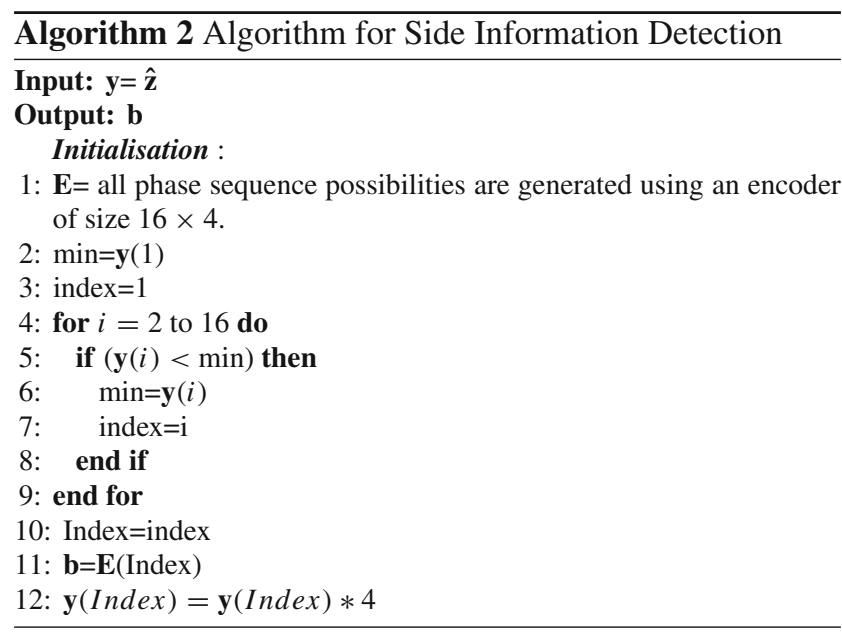

IFFT samples with known factor (by dividing it on $M=4$ )to give the minimum power among the first of 16 samples of OFDM symbol.

\subsection{Receiver side of the proposed technique}

At the receiver side, we applied the detection schemes in [4] but with $M=4$, as explained in algorithm 2, there is an encoder that is similar to that at the transmitter. The first 16 samples of the OFDM symbol are tested to determine the least sample power among them, identify its index, and insert this index into the encoder to generate the phase sequence. For example, if the index of the sample giving the minimum power is 15 , then the input of encoder number 15 will be $\mathrm{ON}$, and its output will be $\{1110\}$. Thus, this technique obviate the sending of side information.

In the proposed technique, the objective is to determine the time domain signal to be added to the original time domain signal $\mathbf{x}$ after phase rotation, to reduce the PAPR. Thus, the data vector changes to $\mathbf{X}+\mathbf{C}$ after the scrambling process, which results in new modulated OFDM signals.

At the receiver, we need to remove the effect of $\mathbf{C}$ at the receiver. In this technique, the PAPR reduction signal is constructed as follows: 
$\mathbf{y}=\hat{\mathbf{z}}$

Thus, the input signal of fast Fourier transforms (FFT) $\hat{x}$ is reconstructed, first, by partitioning the received signal $y$ into four partitions as follows:

$\mathbf{y}_{1}=y_{0, \ldots, k-1}$,

$\mathbf{y}_{2}=y_{k, \ldots, 2 k-1}$,

$\mathbf{y}_{3}=y_{2 k, \ldots, 3 k-1}$,

$\mathbf{y}_{4}=y_{3 k, \ldots, 4 k-1}$

where $k=(N / 4)$. Then, the signal $c$ is generated in the same manner at the transmitter and partitioned into four partitions, as follows:

$\mathbf{c}_{\mathbf{0}}=c_{0, \ldots, k-1}$,

$\mathbf{c}_{\mathbf{1}}=c_{k, \ldots, 2 k-1}$,

$\mathbf{c}_{2}=c_{2 k, \ldots, 3 k-1}$,

$\mathbf{c}_{3}=c_{3 k, \ldots, 4 k-1}$

Afterward, if the phase sequence is $\left\{b_{1}, b_{2}, b_{3}, b_{4}\right\}$, the input signal of FFT $\hat{x}$ can be expressed as follows:

$$
\begin{aligned}
& \hat{x}_{0, \ldots, k-1}=\left(\mathbf{y}_{\mathbf{1}}-\left(\mathbf{c}_{\mathbf{2}} * \hat{b}_{4}\right)\right) * \hat{b}_{1}, \\
& \hat{x}_{k, \ldots, 2 k-1}=\left(\mathbf{y}_{\mathbf{2}}-\left(c_{\mathbf{3}} * \hat{b}_{4}\right)\right) * \hat{b}_{2}, \\
& \hat{x}_{2 k, \ldots, 3 k-1}=\left(\mathbf{y}_{3}-\left(\mathbf{c}_{\mathbf{0}} * \hat{b}_{1}\right)\right) * \hat{b}_{3}, \\
& \hat{x}_{3 k, \ldots, 4 k-1}=\left(\mathbf{y}_{\mathbf{4}}-\left(c_{\mathbf{1}} * \hat{b}_{1}\right)\right) * \hat{b}_{4}
\end{aligned}
$$

where $\hat{b}_{m}=(-1)^{b_{m}}$.

When the reconstructed signal $\hat{x}$ passes through the FFT block, the N-point FFT output is obtained as follows:

$\hat{\mathbf{X}}=\left[\hat{X}_{0}, \hat{X}_{1}, \ldots, \hat{X}_{N-1}\right]^{T}$

\section{Computational complexity}

Complexity associated with the number of complex multiplications and complex additions is related to the number of iterations, which is only 4 in the case of our proposed technique, so clearly this is the lowest among other lowcomplexity multiplicative PAPR reduction techniques.

PTS and TR techniques are unsuitable and expensive for real hardware implementation. This is because, in PTS, it is necessary to do a comprehensive search to identify the optimal phase factors and also because of the need to perform computation and comparison of the PAPR for $W^{M-1}$ candidate phase sequences, where $W$ is the number of phase weight factors. In TR, complexity arises because of the necessity to do a lot of iterations to generate the best peak-canceling signal, which is generated by using $Q$-PRTs. The computational complexity for both techniques is too high when $N$ is large.

In C-PTS, the total complexity [2] for oversampling factor of $L=1$ can be given by

$T_{C-P T S}=3 M N / 2 \log _{2} N+2 M N W^{M-1}$

As shown in (27), the most significant factors that contribute to the complexity of the C-PTS technique are the $M$ - IFFT blocks, the $N$ - point IFFT, and the calculation and comparison of $W^{M-1}$ different PAPRs. In our proposed technique, there are only two-phase weight factors, $\{0,1\}$ and the calculation and comparison of PAPRs is performed only among $M=4$ candidate phase sequences. These adaptations reduce the total complexity to:

$T_{\text {Proposed-Tech. }}=3 M N / 8 \log _{2} N+M N(M / 2)$

From the first term of (28), it is evident that the complexity of the IFFT itself does not change. However, the complexity of the search algorithm, represented by the second term of (28) is significantly reduced.

A measure of the complexity reduction of the proposed technique against the C-PTS, called the computational complexity reduction ratio (CCRR), can be defined as

$C C R R=\left(1-\frac{\text { Complexity of proposed technique }}{\text { Complexity of C_PTS tech. }}\right) \times 100$

Table 2 presents a comparison of the CCRRs of the C-PTS technique [3], the Optimal Search [11], and our proposed technique for $M=4, L=4, W=2$, and $N=512$. Since the complexity associated with the number of complex multiplication and addition operations is dependent on the number of iterations, the number of iterations is listed and considered in Table 2. This table illustrates that compared with the CPTS technique, the Optimal Search gives a CCRR of $50 \%$, whereas our proposed technique achieves a CCRR of $75 \%$. Clearly, our proposed technique offers the lowest computational complexity among the available low complexity PTS techniques.

A measure of the complexity reduction of the proposed technique against the new existing PTS, can be defined as

$C C R R=\left(1-\frac{\text { Complexity of proposed technique }}{\text { Complexity of New_PTS tech. }}\right) \times 100$

Based on (30), Table 3 is presented to provide a comparison of CCRRs of the parallel Tabu search algorithm (Parallel TS-PTS) scheme described in [12], artificial bee 
Table 2 CCRRs of the proposed technique compared to other PTS techniques proposed technique Compared to new existing PTS techniques
Table 3 CCRRs of the

\begin{tabular}{lllcl}
\hline PTS schemes & Iterations & Complex add. & $\mathrm{M}=4, \mathrm{~N}=512$ & CCRR (\%) \\
\hline C- PTS & $U=W^{M-1}$ & $N(M-1) U$ & 12,288 & 0 \\
Optimal Search & $U=\left(W^{M-1}\right) / 2$ & $N(M-1) U / 2$ & 6144 & 50 \\
proposed technique & $\mathrm{U}=\mathrm{M}$ & $N(M-1) U / 2$ & 3072 & 75 \\
\hline
\end{tabular}

\begin{tabular}{lllll}
\hline PTS schemes & Iterations & Complex add. & $\mathrm{M}=4, \mathrm{~N}=512$ & CCRR $(\%)$ \\
\hline Parallel TS-PTS & $T 1$ & $N(M-1) T 1$ & 4608 & 0 \\
proposed technique & $\mathrm{M}$ & $N(M-1) M / 2$ & 3072 & 33.33 \\
ABC-PTS & $T 2$ & $N(M-1) T 2$ & 4,608 & 0 \\
proposed technique & $\mathrm{M}$ & $N(M-1) M / 2$ & 3072 & 33.33 \\
SLS-PTS & $T 3$ & $N(M-1) T 3$ & 4,608 & 0 \\
proposed technique & $\mathrm{M}$ & $N(M-1) M / 2$ & 3072 & 33.33 \\
\hline
\end{tabular}

colony algorithm (ABC-PTS) scheme described in [13], and successive local search using sequences (SLS) scheme described in [14] with our proposed technique when $M=$ $16, L=4, W=2, N=512, T 1=3, T 2=3$, and $T 3=P_{0}+(W-1) \sum_{m=1}^{M-1} P_{m}=3$. The number of iterations is considered in Table 3 since the complexity associated with the number of complex multiplication and complex addition respectively, is dependent on the number of iterations. This table shows that proposed technique achieves a CCRR of $33.33 \%$ compared against the CCRR of the Parallel TS-PTS; $33.33 \%$ compared against the ABC-PTS scheme and $33.33 \%$ compared against the SLS scheme. Clearly, our proposed technique achieves the lowest computational complexity among all the compared low complexity PTS schemes.

Unlike the TR, the proposed technique does not need any iteration to generate the best peak-cancelling signal.

\section{Numerical results}

Simulations were conducted using MATLAB to evaluate and compare the performance of the proposed technique with the C-PTS technique, and the original OFDM. We adopted 16-QAM signaling with various IFFT lengths of $N=128$, $256,512, W=2$, and oversampling factor of $L=4$. To obtain the CCDF, $10^{5}$ random OFDM symbols were generated. The CCDFs of the proposed technique, C-PTS and the original OFDM for various numbers of subcarriers $N=$ $\{128,256,512\}$ are presented in Fig. 4. The range of PAPR reduction achieved by adopting proposed technique is $2.7 \mathrm{~dB}$ up to $3.2 \mathrm{~dB}$ compared to original OFDM. From the simulation results shown, shorter IFFT length will achieve higher PAPR reduction compared to longer IFFT length where in this case, for $N=128$ the reduction is $3.2 \mathrm{~dB}$ while for $N=$ 512 IFFT length the reduction is only $2.7 \mathrm{~dB}$. Apart from

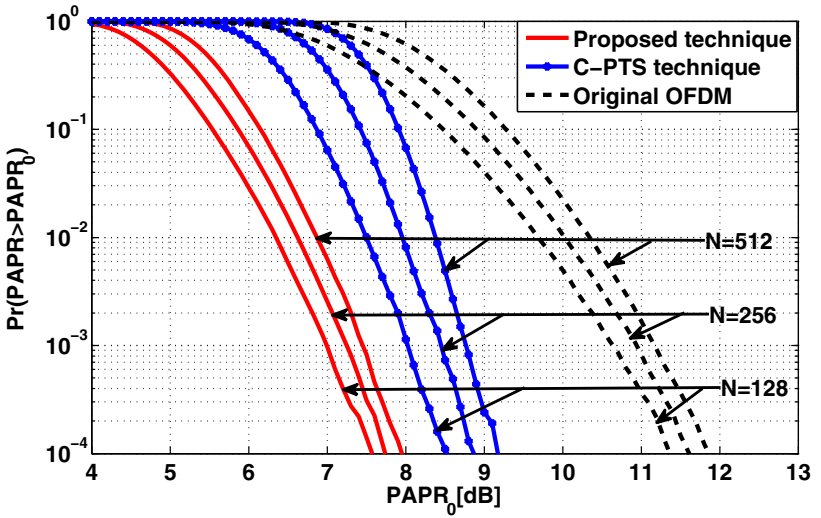

Fig. $4 \mathrm{CCDF}$ of PAPR of the proposed technique for different $N$ subcarriers compared with the C-PTS and original OFDM for $M=4$, $L=4$

that, a comparison between PAPR reduction performance of C-PTS and proposed technique was carried out to determine the ability of proposed technique to decrease PAPR of an OFDM signal. Fig. 4 shows that the proposed technique outperforms C-PTS as the PAPR reduction is higher compared to that of C-PTS technique. The PAPR reduction achievement ranges from $1.14 \mathrm{~dB}$ to $1.18 \mathrm{~dB}$. Among the various IFFT lengths adopted, 128 IFFT lengths achieved the highest improvement which is as much as $1.18 \mathrm{~dB}$. It is evident that the proposed technique yields $2.7-3.2 \mathrm{~dB}$ reduction in the PAPR with respect to the original OFDM transmission with only 4 iterations at a CCDF of $10^{-4}$.

Figure 5 shows a comparison in PAPR reduction performance between the proposed technique and TR technique. From this figure, it is evident that the PAPR reduction of the proposed technique is slightly better compared to that of TR technique. For example, with the TR technique, the proposed technique can achieve $2.63 \mathrm{~dB}, 2.32 \mathrm{~dB}, 1.21 \mathrm{~dB}, 1.96 \mathrm{~dB}$ 


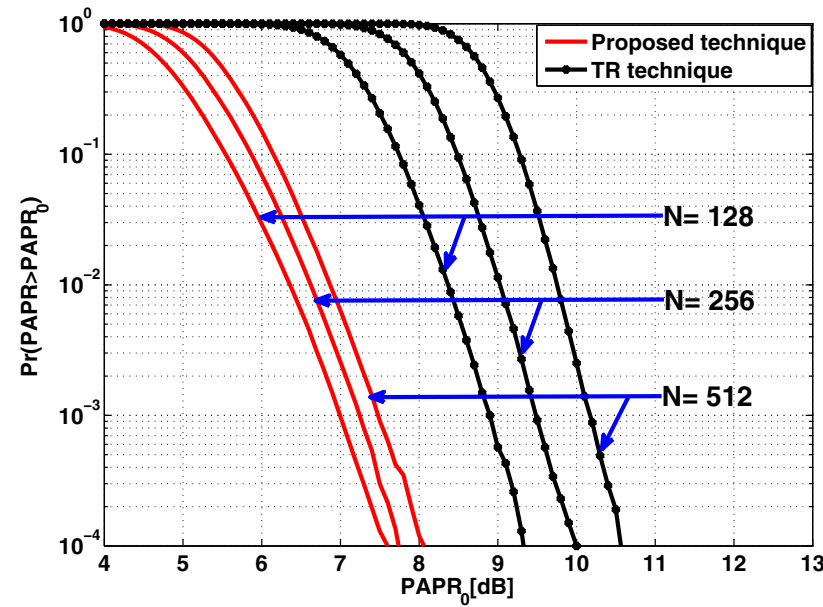

Fig. 5 CCDF of PAPR of the proposed technique for different $N$ subcarriers compared with the TR

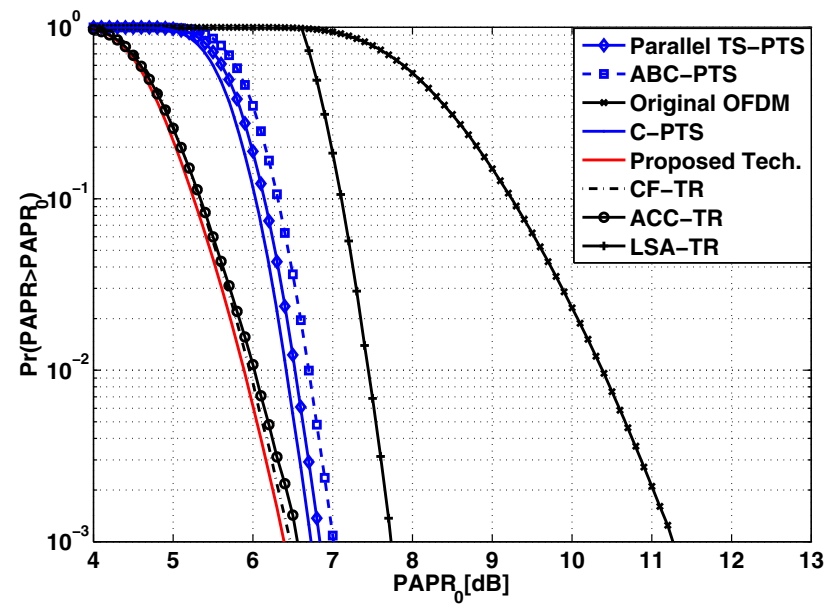

Fig. $6 \mathrm{CCDF}$ of PAPR of the proposed technique and new existing PTS, TR techniques with 16-QAM and $N=256$

PAPR reduction when $N=\{512,256,128\}$ at a CCDF of $10^{-4}$, respectively.

Figure 6 shows a comparison in PAPR reduction performance between the proposed technique, original OFDM, C-PTS, Parallel TS-PTS and ABC-PTS for $M=16, W=2$, $N=256$ and 16-QAM modulation. As shown in this figure, the PAPR of the proposed technique at $\mathrm{CCDF}=10^{-3}$ is $6.39 \mathrm{~dB}$. Meanwhile, the PAPRs of original OFDM, C-PTS, Parallel TS-PTS and ABC-PTS at $\mathrm{CCDF}=10^{-3}$ are 11.24 $\mathrm{dB}, 6.72 \mathrm{~dB}, 6.84 \mathrm{~dB}$, and $7.01 \mathrm{~dB}$, respectively. Compared with C-PTS and new existing PTS techniques, the proposed technique shows better PAPR reduction performance. Figure 6 also shows comparison in PAPR reduction performance between the proposed technique and LSA-TR [15], CF-TR [16] and ACC-TR [17] where the number of the reserved tones $Q=32, N=256$ and 16-QAM modulation. As shown in this figure, the PAPR of the proposed technique

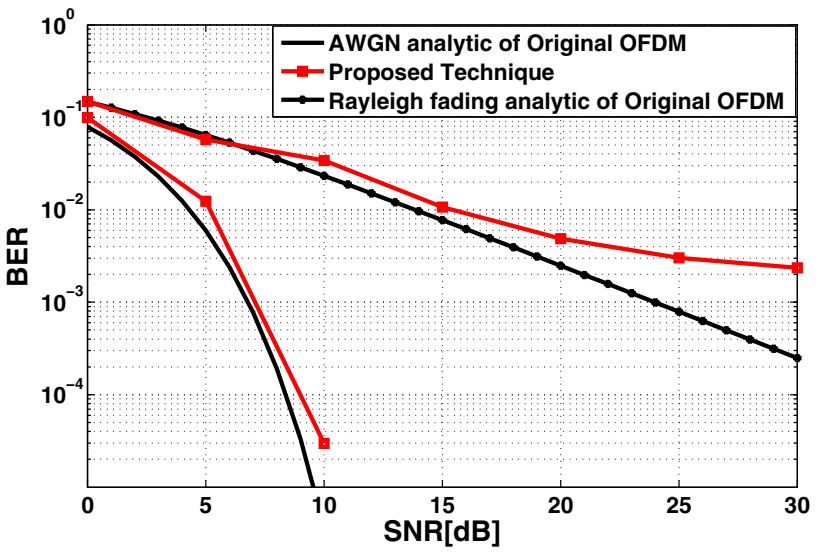

Fig. 7 BER performance for OFDM system and proposed technique with 4-QAM and $N=256$

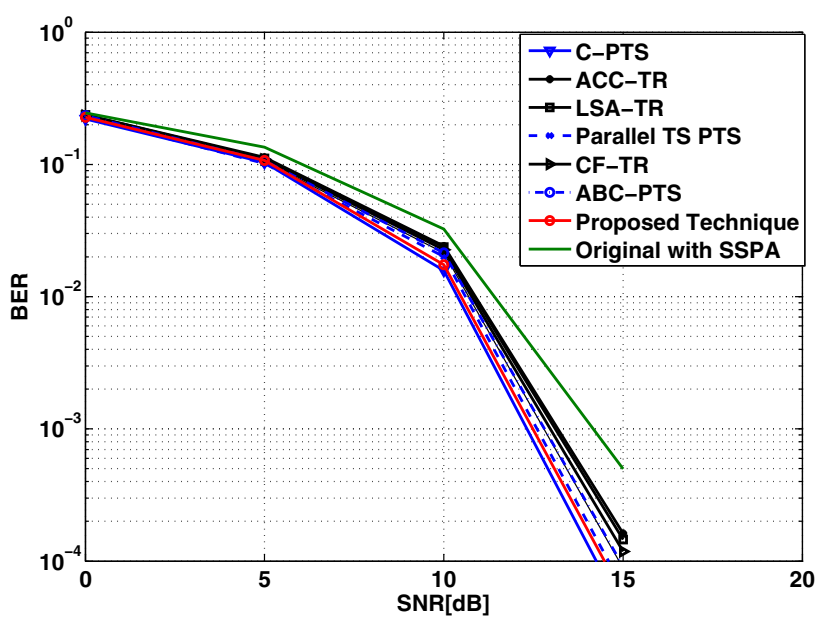

Fig. 8 Comparison of BER performance of the proposed technique and new existing PTS, TR techniques with 4-QAM and $N=256$

at $\mathrm{CCDF}=10^{-3}$ is $6.39 \mathrm{~dB}$. Meanwhile, the PAPRs of LSA$\mathrm{TR}, \mathrm{ACC}-\mathrm{TR}$ and $\mathrm{CF}-\mathrm{TR}$ at $\mathrm{CCDF}=10^{-3}$ are $7.73 \mathrm{~dB}, 6.56$ $\mathrm{dB}$, and $6.74 \mathrm{~dB}$, respectively. Compared with new existing TR techniques, the proposed technique shows better PAPR reduction performance.

In Fig. 7, the analytical BER expressions for M-ary QAM signaling in additive white gaussian noise (AWGN) and multipath Rayleigh fading channel [18] are respectively given as:

$$
\begin{aligned}
& P_{e}=\frac{2(U-1)}{M \log _{2} U} Q\left(\sqrt{\frac{6 E_{b}}{N_{o}} \cdot \frac{\log _{2} U}{U^{2}-1}}\right) \\
& P_{e}=\frac{U-1}{U \log _{2} U}\left(1-\sqrt{\frac{3 \gamma \log _{2} U /\left(U^{2}-1\right)}{3 \gamma \log _{2} U /\left(U^{2}-1\right)+1}}\right)
\end{aligned}
$$

where $\gamma$ and $U$ denote $E_{b} / N_{\circ}$ and the modulation order, respectively, while $Q(\cdot)$ is the standard Q-function defined 
as

$Q(\cdot)=\frac{1}{\sqrt{2 \pi}} \int_{x}^{\infty} e^{-t^{2} / 2} d x$

We utilized 4-QAM signaling with IFFT length of 256 in order to obtain BER performance in AWGN and multipath Rayleigh fading channel (with the maximum delay of 15 samples). The results are presented in Fig. 7. In Fig. 7, the performance bounds are obtained by ignoring the effect of the high power amplifier (HPA) and directly transmitting the OFDM signals through AWGN and Rayleigh fading channels. Notably, BER performance in AWGN channel and the Rayleigh fading channel is consistent with the analytical result.

Figure 8 shows the BER performance with employing the Rapps solid-state power amplifier (SSPA) HPA model with the nonlinearity parameter to be 2 and the input backoff (IBO) to be $0 \mathrm{~dB}[19,20]$ for $M=4, W=2, N=256$ and 4-QAM modulation. This figure shows a comparison of BER performance of the proposed technique, original OFDM, C-PTS, Parallel TS-PTS, ABC-PTS, LSA-TR, ACC-TR and CF-TR over AWGN channel. As shown in this figure, the proposed technique has the best BER performance over the original OFDM and new existing PTS and TR techniques at BER $=10^{-4}$. Compared with C-PTS, the proposed technique offer nearly same BER performance as that of the C-PTS technique.

\section{Conclusion}

We proposed a novel hybrid $\mathrm{CF}$ reduction technique for OFDM systems with no side information and a low computational requirement that results in low complexity. A new partitioning and optimization schemes are established. In optimization scheme, only a two phase sequence is required with only four iterations. Thus, compared to the other PAPR reduction techniques is considered unique while giving superior performance.

Acknowledgements This work was supported by Universiti Putra Malaysia under the Prototype Development Research Grant Scheme (PRGS) of the Ministry of Higher Education, Malaysia. (No. 5528700).

\section{References}

1. Rahmatallah, Y., \& Mohan, S. (2013). Peak-to-average power ratio reduction in OFDM systems: A survey and taxonomy. IEEE Communications Surveys and Tutorials, 15(4), 1567-1592.

2. Varahram, P., \& Ali, B. M. (2011). Partial transmit sequence scheme with new phase sequence for PAPR reduction in OFDM systems. IEEE Transactions on Consumer Electronics, 57(2), 366371.
3. Müller, S. H., \& Huber, J. B. (1997). OFDM with reduced peakto-average power ratio by optimum combination of partial transmit sequences. Electronics Letters, 33(5), 368-369.

4. Al-Hussaini, K., Ali, B. M., Varahram, P., Hashim, S., \& Farrell, R. (2016). A new subblocks interleaving PTS Technique with minimum processing time for PAPR reduction in OFDM systems. Electronics Letters, 1(1), 21. doi:10.1049/joe.2016.0074.

5. Jiang, T., \& Wu, Y. (2008). An overview: Peak-to-average power ratio reduction techniques for ofdm signals. Electronics Letters, 54(2), 257268

6. Al-Hussaini, K. and Ali, B. M. and Varahram, P. and Hashim, S.K. Al-Hussaini, B. M. Ali, P. Varahram, S. Hashim (2016). A novel low complexity high efficiency hybrid PAPR reduction for OFDM systems. 2015 IEEE 12th Malaysia International Conference on Communications (MICC) (MICC'15), p. 345350.

7. Sabbir, A., \& Makoto, K. (2013). Interleaving effects on BER fairness and PAPR in OFDMA System. IEEE Communications Letters, 52,18393

8. Mukunthan, P., \& Dananjayan, P. (2014). Modified PTS with Interleaving for PAPR Reduction of OFDM Signal with QPSK Subblock. Electronics Letters, 3, 1-8.

9. Han, S. H., \& Lee, J. H. (2005). An overview of peak-to-average power ratio reduction techniques for multicarrier transmission. Electronics Letters, 12(2), 56-65.

10. Goldsmith, A. (2005). Wireless communications, chap. 12. Cambridge: Cambridge University Press.

11. Alavi, A., Tellambura, C., \& Fair, I. (2005). PAPR reduction of OFDM signals using partial transmit sequence: An optimal approach using sphere decoding. IEEE Communications Letters, 9(11), 982-984.

12. Taspinar, N., Kalinli, A., \& Yildirim, M. (2011). Partial transmit sequences for PAPR reduction using parallel tabu search algorithm in OFDM systems. IEEE Communications Letters, 15(9), 974-976.

13. Wang, Y., Chen, W., \& Tellambura, C. (2010). A PAPR reduction method based on artificial bee colony algorithm for OFDM signals. IEEE Communications Letters, 9(10), 2994-2999.

14. Cho, Y., No, J., \& Shin, D. (2012). A new low-complexity PTS scheme based on successive local search using sequences. IEEE Communications Letters, 16(9), 1470-1473.

15. Li, H., Jiang, T., \& Zhou, Y. (2011). An improved tone reservation scheme with fast convergence for papr reduction in ofdm systems. IEEE Communications Letters, 57(4), 902906.

16. Jiang, T., Ni, C., Xu, C., \& Qi, Q. (2014). Curve fitting based tone reservation method with low complexity for papr reduction in ofdm systems. IEEE Communications Letters, 18(5), 805808.

17. Wang, Y., Chen, W., \& Tellambura, C. (2012). Genetic algorithm based nearly optimal peak reduction tone set selection for adaptive amplitude clipping papr reduction. IEEE Communications Letters, 58(3), 462471.

18. Proakis, J. G., \& Masoud, S. (2008). Digital Communications 5/E. New York: McGraw-Hill.

19. Krongold, B. S., \& Jones, D. (2004). An active-set approach for ofdm par reduction via tone reservation. IEEE Communications Letters, 52(2), 495509.

20. Krongold, B. S., \& Jones, D. (2003). Par reduction in ofdm via active constellation extension. In 2003 IEEE international conference on acoustics, speech, and signal processing (ICASSP 03), Vol. 4, 52, p.IV5258. 


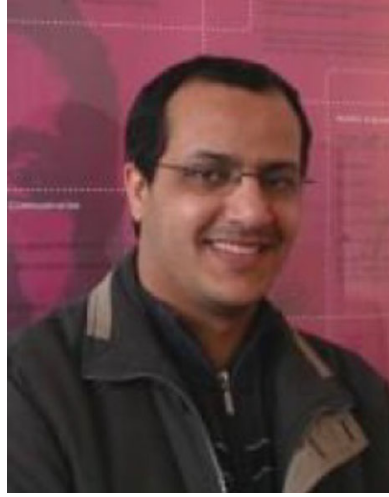

Khalid Al-Hussaini received his B.Sc. Information Engineering from Baghdad University, Iraq in 2003, his M.Sc. Electrical Communication Engineering from Kassel University, Germany in 2010. Currently, he is pursuing his Ph.D. in Wireless Communication Engineering in the University Putra Malaysia (UPM), Malaysia. His research interests are multiple-input multipleoutput (MIMO) systems, OFDM and Multi-carrier systems, PAPR reduction in OFDM systems, Linearization of power amplifiers and FPGA design.

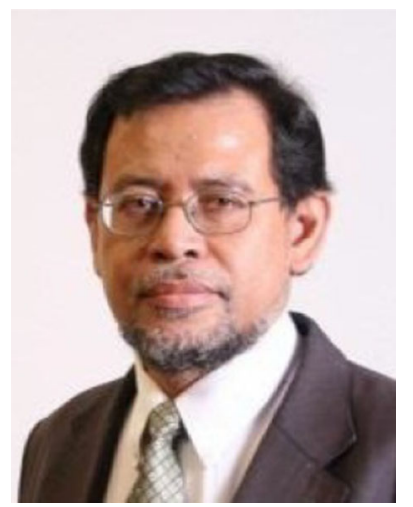

Borhanuddin M. Ali graduated from Loughborough University, UK, in 1979 and M.Sc. and $\mathrm{Ph}$.D. from University of Wales, Cardiff, in 1981 and 1985 respectively. Upon his Ph.D. graduation, he joined Universiti Pertanian Malaysia (now renamed Universiti Putra Malaysia) as a lecturer, and became Professor in 2002, during which he served as Head of Departments, twice, and later the Director of Institute of Multimedia and Software, a centre of excellence within the same university. Over his tenureship, he also spent times with the industry, first with Celcom R\&D (a cellular telco) in 1995, then with Might (a government industry group on high tech), 1997-98; and more recently, with Mimos (a government research lab in ICT), 2006-2008. He is also active in professional societies; serving as Chair of ComSoc Chapter Malaysia, twice, and then as the Chair of IEEE Malaysia Section 2002-2004. He also serves as committee member of various portfolios at ComSoc AP Board, and in the steering committee of APCC. He is currently the Director of the Institute of Advanced Technology, a university centre of excellence in engineering, science and IT. His research interest spans the broad area of wireless communications and networks technology.

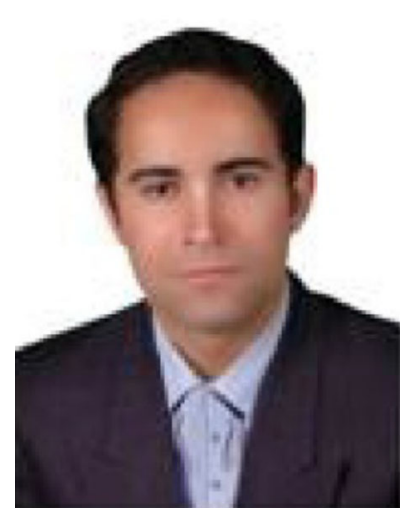

Pooria Varahram received his B.Sc. Electrical and Electronics Engineering from Khaje Nasir University of Technology in 2002, his M.Sc. Telecommunications Engineering from Tarbiat Modares University in 2004 and Ph.D. in Wireless Communication Engineering from the University Putra Malaysia (UPM) in 2010. He has more than 5 years experience in designing and developing a range of electronic and telecommunication related projects. His research interests are PAPR reduction in OFDM systems, Linearization of power amplifiers, microwave power amplifiers design.

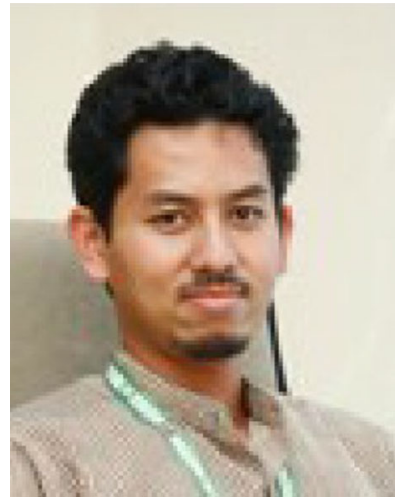

network security.

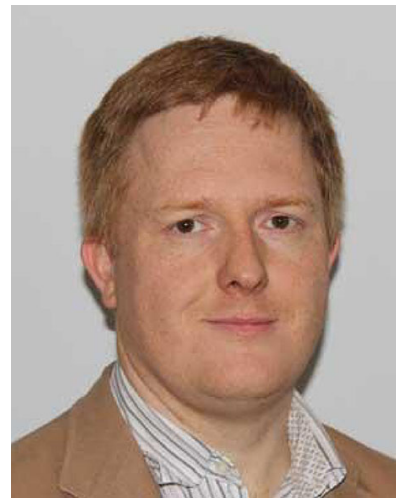

Shaiful Jahari Hashim is currently a senior lecturer in Department of Computer and Communication Systems Engineering, Faculty of Engineering, Universiti Putra Malaysia. He received his $\mathrm{Ph} . \mathrm{D}$ from Cardiff University, UK (2011), M.Sc from Universiti Kebangsaan Malaysia (2003) and B.Eng from University of Birmingham, UK (1998) in the field of Electrical and Electronics Engineering. His research interests are energy-efficient wireless system, cloud computing and

Ronan Farrell received his B.E. and Ph.D. from University College Dublin in 1993 and 1998 respectively. He worked with ICI/Zeneca Chemicals from 1993-1995 and Parthus Technologies from 1998-2001 as a mixed signal ASIC designer. In 2001 he joined Maynooth University as a lecturer, promoted to Senior Lecturer in 2010. In 2008 he was appointed Director for the Callan Institute for Applied ICT, and in 2012 was named Head of the Department of Electronic Engineering. In 2004, he became an SFI theme leader for radio frequency electronics research within the Centre for Telecommunications Value-Chain Driven Research (CTVR) and remains in that position. In 2008 he became a strand co-leader for sensors networks in an SFI Cluster on advanced Geotechnologies with a focus on wide area wireless sensor networks. Ronan has published over a hundred peer-reviewed papers. He holds three patents and licensed technology that has led to the spin-out three companies, Ronans personal research interests include wireless system design, electronics and radio systems. 